\title{
Plasma cytokine profiles associated with rhodesiense sleeping sickness and falciparum malaria co-infection in North Eastern Uganda
}

\author{
Julius Nsubuga ${ }^{1 *} \mathbb{D}$, Charles Drago Kato ${ }^{1}$, Ann Nanteza ${ }^{1}$, Enock Matovu ${ }^{1}$ and Vincent Pius Alibu²
}

\begin{abstract}
Background: Immunological Human African Trypanosomiasis (HAT) studies often exclude malaria, although both infections overlap in specific endemic areas. During this co-infection, it is not known whether this parasitic interaction induces synergistic or antagonistic cytokine response among humans. This study determined prevalence of Plasmodium falciparum malaria among Trypanosoma brucei rhodesiense HAT and plasma cytokine profile levels associated with HAT and/or malaria infections.
\end{abstract}

Methods: Participants were recruited at Lwala hospital in north eastern Uganda: healthy controls (30), malaria (28), HAT (17), HAT and malaria (15) diagnosed by microscopy and PCR was carried out for parasite species identification. Plasma cytokine levels of Interferon-gamma (IFN- $\gamma$ ), Tumour Necrosis Factor-alpha (TNF-a), Interleukin (IL)-6, IL-10 and Transforming Growth Factor-beta (TGF- $\beta$ ) were measured by sandwich Enzyme-Linked Immuno Sorbent Assay and data statistically analysed using Graphpad Prism 6.0.

Results: The prevalence of P. falciparum malaria among T. rhodesiense HAT cases was high (46.8\%). Malaria and/or HAT cases presented significant higher plasma cytokine levels of IFN- $\gamma$, TNF- $\alpha$, IL-6, IL-10 and TGF- $\beta$ than healthy controls $(P<0.05)$. Levels of IFN- $\gamma$, IL- 6 and IL-10 were significantly elevated in HAT over malaria $(P<0.05)$ but no significant difference in TNF- $\alpha$ and TGF- $\beta$ between HAT and malaria $(P>0.05)$. Co-infection expressed significantly higher plasma IFN- $\gamma, I L-6$, and IL-10 levels than malaria $(P<0.05)$ but no significant difference with HAT mono-infection $(P>0.05)$. The TNF-a level was significantly elevated in co-infection over HAT or malaria mono-infections $(P<0.05)$ unlike TGF- $\beta$ level. Significant positive correlations were identified between IFN- $\gamma$ verses TNF- $\alpha$ and IL-6 verses IL-10 in co-infection (Spearman's P <0.05).

Conclusions: The T. b. rhodesiense significantly induced the cytokine response more than $P$. falciparum infections. Co-infection led to synergistic stimulation of pro-inflammatory (IFN- $\gamma$, TNF-a), and anti-inflammatory (IL-6, and IL-10) cytokine responses relative to malaria mono-infection. Level of TNF-a partially indicates the effect induced by T. $b$. rhodesiense and P. falciparum mono-infections or a synergistic interaction of co-infections which may have adverse effects on pathogenesis, prognosis and resolution of the infections.

Trial registration VCD-IRC/021, 26/08/2011; HS 1089, 16/01/2012

Keywords: HAT, Malaria, Co-infection, Mono-infection, Cytokine, IFN- $\gamma$, TNF- $a$, IL-6, IL-10, TGF- $\beta$

\footnotetext{
${ }^{*}$ Correspondence: joeljuliusnsubuga@yahoo.com

${ }^{1}$ College of Veterinary Medicine, Animal Resources \& Bio-security,

Makerere University, Kampala, Uganda

Full list of author information is available at the end of the article
} 


\section{Background}

Human African Trypanosomiasis (HAT) and/or malaria are two protozoan parasitic vector borne diseases of public health concern in endemic areas of Africa. In Uganda, malaria is prevalent throughout the country whereas HAT is endemic in specific foci. Nevertheless, malaria coincides with HAT in specific overlapping areas. Therefore plasmodium and trypanosome infection may exist within a host concurrently $[1,2]$. This is because tsetse flies and mosquitoes that transmit HAT and malaria parasites respectively, share these specific co-endemic habitats [3]. This co-infection is of epidemiological and immunological importance, although most studies focus on single-pathogen infections. Studies conducted in East Africa showed malaria prevalence among HAT cases of $28.9 \%$ in eastern Uganda [2], 2.9\% in Uganda, 79.7\% in Tanzania [1], and $100 \%$ in Kenya [3]. Also, HAT cases present clinical symptoms similar to those of malaria [4]. This makes diagnosis and management of both diseases difficult in overlapping areas. Nevertheless, HAT clinical symptoms and fatality are not significantly affected by malaria $[1,2]$.

At immunological level, quite different immune cytokine responses are induced by different parasites. Studies in animal and human co-infections suggest significant variation in the immune cytokine response to occur in reaction to pathogen-pathogen interactions. This is essential in the pathogenesis of infections (pathogen-host interactions) and their interactions (pathogen-pathogen interactions) in the host. During co-infections, the parasites may exhibit synergistic, antagonistic, or competitive interactions with the immune system of host $[5,6]$. The proinflammatory or anti-inflammatory cytokines secreted may cause cross-immunity or immune-suppression leading to partial host protection and regulation of infections. When these parasitic interactions induce immunosuppression, it enhances parasite survival and pathogenicity in the host [5]. Cytokine modulation is thought to be associated with the degree of clinical manifestation, parasitemia, pathogenesis, disease severity and survival among HAT [7-9] or malaria cases [10-12]. The pro-inflammatory cytokines (like IFN- $\gamma$ ) are important in parasite control during early stage infection by activation of TNF- $\alpha$ and nitric oxide (NO) secretion from macrophages, and then a switch to anti-inflammatory cytokines (IL-10, IL-4) with protective immunity crucial for survival of host or parasite during late and chronic stages of trypanosomiasis [13, 14]. Similarly pro-inflammatory cytokines are essential in the resolution of parasitemia, clinical manifestation and pathogenesis, especially during the early stages of $P$. falciparum infection [11,
12, 15]. Anti-inflammatory cytokines like TGF- $\beta$ are produced to regulate pro-inflammatory responses and secretion [16]. Also identified for its anti-inflammatory protective role in autoimmune conditions [17], IL-10 act as an immunoregulator neutralizing the effects of inflammatory responses associated with immunopathology and severe forms of $P$. falciparum infection [10, 18]. In animal models, IFN- $\gamma$ level was described to be more elevated in $T$. brucei than Plasmodium berghei infected mice. Co-infected mice expressed elevated IFN- $\gamma$ and TNF- $\alpha$ levels over $P$. berghei or T. brucei mono-infected group suggesting active response against secondary infection. Although IFN- $\gamma$ in co-infected mice was more less than in $T$. brucei mono-infected group. The induction towards pro-inflammatory response (TNF- $\alpha$, IFN- $\gamma$ and NO) by $T$. brucei could account for plasmodium hepatic impairment in mice [19]. However, anti-inflammatory IL-10 plasma level was significantly lower in $T$. $b$. gambiense and $P$. falciparum co-infection than healthy controls. Consequently, IL-10 plasma level of HAT was similar irrespective of $P$. falciparum infection [20].

The plasmodium and trypanosome infections have been extensively described separately with potential to induce cytokine production in the host. Despite epidemiological HAT and malaria co-infection studies, immunological cytokine response to $T$. $b$. rhodesiense and $P$. falciparum co-infection, and its relative comparison to mono-infection in naturally infected cases are not or poorly explored. Immunological HAT studies have always excluded malaria although both infections overlap in specific co-endemic areas of tropical Africa $[8,10]$. It is not known whether this parasitic interaction induces synergistic or antagonistic cytokine response among co-infected humans relative to either mono-infection. The study determined prevalence of $P$. falciparum malaria among T. $b$. rhodesiense HAT cases and plasma cytokine profile levels associated with parasitological acute sleeping sickness and/or malaria cases from north eastern Uganda. The results will provide insights that can be manipulated in future to aid clinicians, diagnostic approaches, vector or disease control policy team on how to handle HAT and malaria cases. The comparison of cytokine concentration examined how $T . b$. rhodesiense HAT and P. falciparum malaria co-infection modify the immunological cytokine response induced by the two parasites relative to monoinfections. This will contribute to the understanding of the immunological response of this co-infection and management of cases, emphasising the significance of immune-mediated interactions in poly-parasitism among people. 


\section{Materials and methods}

\section{Study area}

Participants were recruited from north eastern Uganda at Lwala hospital, a sleeping sickness referral center in Kaberamaido district, providing health services especially to HAT cases. Since 2004, this area has been affected by $T$. $b$. rhodesiense HAT which extended from the historical foci in the eastern part of the country [21]. Currently, HAT has been identified to be prevalent from a large endemic area of Dokolo, Kaberamaido, Soroti, Lira, Alebtong, and Kole districts (Fig. 1). Within this area, an approximation of 7.9 million people are at risk of acquiring T. $b$. rhodesiense sleeping sickness [22]. The highest occurrence of the disease has been noted in Dokolo and Kaberamaido with $60.9 \%$ of T. $b$. rhodesiense infected cattle as human reservoirs [23]. Malaria is also prevalent in north eastern Uganda that coincides with HAT [2].

\section{Study design and sample collection}

This was a case control study to determine the prevalence of $P$. falciparum malaria among $T$. $b$. rhodesiense HAT cases and associated plasma cytokine levels of HAT and/or malaria cases from the region reporting to Lwala hospital, aged 6 years and above between October/2013 and May/2014. Patients report to hospital with common complaints of; fever (temperature $>37^{\circ} \mathrm{C}$ ), general body weakness, headache, joint pain, tremor, loss of appetite and sweating. It is a routine practice at Lwala hospital to microscopically check for malaria and HAT parasites. After patients had given detailed clinical descriptions to the medical physician, participants were subjected to routine laboratory diagnostic procedures at the hospital required to guide treatment. Microscopic examination of wet and thick blood films from finger prick blood stained with $10 \%$ Giemsa or Field stain for plasmodium and trypanosomes [4, 24], or haematocrit centrifugation technique (HCT) for trypanosomes [25] were performed. After detection of parasites in participants, blood $(5 \mathrm{ml})$ by venepuncture using EDTA vacutainer tubes (BD-Plymouth, UK) was collected after consent before treatment for HAT and/or malaria. If blood smear was positive for trypanosomes or patient presented with HAT signs (convulsions, tremors, psychotic behaviour and sleep disorders), lumber puncture was performed to obtain CSF (3 ml) following WHO sleeping sickness staging guidelines [22]. White blood cell (WBC) count in CSF was performed by Neubauer Haemocytometer. Analysis of CSF for trypanosomes was executed by the modified single centrifugation method [26]. The presence of trypanosomes in blood but absent in CSF or WBC count $<5$ cells $/ \mathrm{mm}^{3}$ was categorised as early stage HAT while late stage infection was confirmed by the presence of trypanosomes in the CSF and/or WBC count $>5$ cells/ $\mathrm{mm}^{3}$.

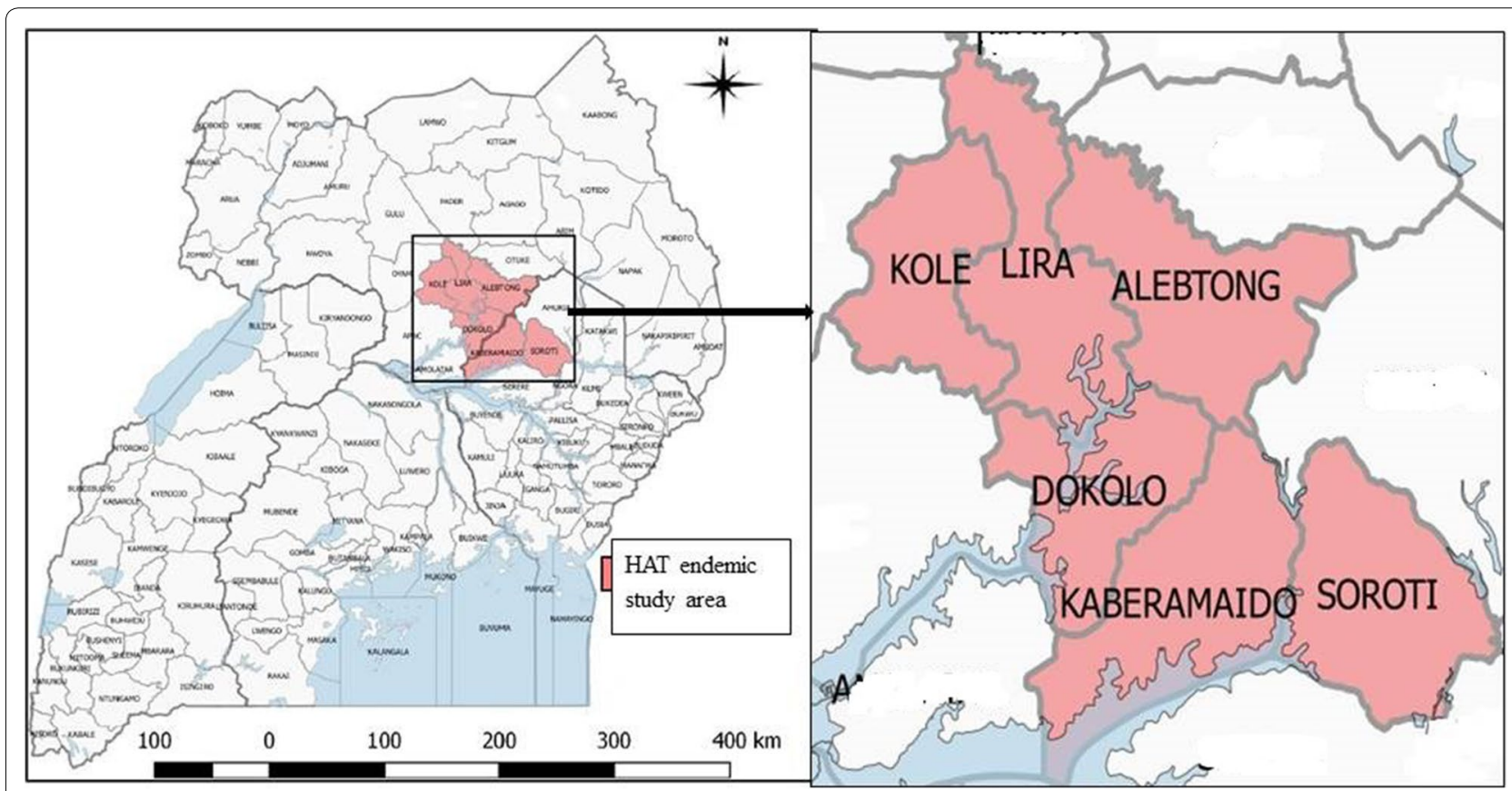

Fig. 1 Map of Uganda showing the six districts with Human African Trypanosomiasis and malaria cases reporting at Lwala hospital. Map of Uganda was sourced from Uganda bureau of statistics [27] and modified. Map of the study area was drawn using QGIS 
Following this diagnosis, patients were recommended for the appropriate treatment and monitoring by the clinician. For HAT, this involved administering Suramin and Melarsoprol for early and late stage HAT respectively [22]. Malaria patients were treated with Artemisininbased combination therapy (ACT) [27]. Healthy controls were mobilised from their homes within the study area by the principal investigator and the field team to the hospital with prior knowledge about the study purpose. The blood collected was subjected to the routine laboratory diagnosis at the hospital. The collected blood $(5 \mathrm{ml})$ from cases and controls was centrifuged for $10 \mathrm{~min}$ at $3000 \mathrm{rpm}$ using Eppendorf centrifuge (5424R, Germany) to obtain plasma and packed cell volume (PCV) of blood. The plasma $(2 \mathrm{ml})$ and PCV $(2 \mathrm{ml})$ were aliquoted and stored in liquid nitrogen. The participant samples were transported to Afrique One laboratory, College of Veterinary Medicine, Animal Resources and Biosecurity (COVAB), Makerere University for SRA or MSP-2 PCR and cytokine analysis. The sample size was calculated using $\mathrm{G}^{*}$ Power 3.0 software giving a total of 90 participants considered for the study. The minimum number per group (HAT-malaria, HAT, malaria and healthy controls) for cytokine analysis was 15 participants (Power $=80 \%$ and $\mathrm{P}=0.05$ ).

\section{Inclusion and exclusion criteria}

All HAT and/or malaria cases and healthy control individuals diagnosed from the region reporting at Lwala hospital were included, after a written informed consent in their local language (Kumam) from adults or guardians in case of minors $(6<18$ years $)$ in addition to their assent. A case was an individual with positive blood smear of trypanosome and/or plasmodium parasites in blood, or presence of trypanosomes in CSF. For parasite species identification, SRA (T. b. rhodesiense) and/or MSP-2 (P. falciparum) nested PCR was carried out. A healthy control was an individual without any detectable parasite on blood smear or other infections and SRA or MSP-2 PCR negative. Individuals diagnosed with infections of helminths (microscopic examination of filtered urine for the presence of eggs), amebiasis (detection of cysts in stool), typhoid (Widal method), tuberculosis (sputum microscopy), human immunodeficiency virus (HIV) (rapid diagnostic test strip) and Urinary tract infections (UTI) (urine test strip compared to coloured scale) [4] were excluded. Children below 6 years were excluded from the study due to their developing and differences in capability of immune system [28]. Previously HAT and/ or malaria treated individuals before a lapse of 1 month to eliminate effect of slow acting drugs [29] and those who did not consent to the study were excluded from study.

\section{Amplification of Trypanosoma brucei rhodesiense serum resistance associated gene}

The extraction of DNA from blood PCV was performed using QIAGEN DNeasy Blood \& Tissue Kit (Qiagen ${ }^{\circledR}$, USA) according to manufacturer's instructions. The eluted DNA was stored at $-20{ }^{\circ} \mathrm{C}$ for PCR analysis. Nested SRA PCR was used to detect $T$. $b$. rhodesiense using sequence primers (Microsynth, Switzerland) on test samples, positive control (Genomic DNA extracted from previously characterised strain of $T$. $b$. rhodesiense from Tororo endemic area) or negative control (PCR water, Bioline, USA) with conditions as described by Maina et al. [30] and products visualized under UV on $2 \%$ gel stained with $0.5 \mu \mathrm{g} / \mathrm{ml}$ ethidium bromide (Sigma, USA).

\section{Amplification of Plasmodium falciparum merozoite surface protein-2 gene}

Nested MSP-2 PCR was used to detect the presence of $P$. falciparum using sequence specific primers ((Biolegio, Netherlands) on test samples, positive control (3D7 or HB3 Genomic DNA extracted from cloned strains, Invitrogen, USA) or negative controls (PCR water, Bioline, USA) with conditions as described by Dokomajilar et al. [31] and products visualized under UV on $2 \%$ gel stained with $0.5 \mu \mathrm{g} / \mathrm{ml}$ ethidium bromide (Sigma, USA).

\section{Plasma cytokine assays}

The selected cytokines (IFN- $\gamma$, TNF- $\alpha$, IL- 6 , IL-10 and TGF- $\beta$ ) have been associated with the pathogenesis, disease severity and survival in either HAT [7-9] or malaria [11, 12, 15]. Plasma concentrations of cytokines (IFN- $\gamma$, TNF- $\alpha$, IL-6, IL-10, and TGF- $\beta$ ) were assayed using solid phase sandwich ELISA kit (BD OptEIA ${ }^{\mathrm{TM}}$, San Diego, USA) as described previously [8, 32]. Briefly, 96 well microplates (nunc ${ }^{\mathrm{TM}}$, Denmark) were coated with $100 \mu \mathrm{l}$ per well of capture antibody diluted in coating buffer $(1 \times$ phosphate-buffered saline, PBS) and incubated over night at $4{ }^{\circ} \mathrm{C}$ (Electrocool LG, South Korea). Microplates were aspirated and washed 3 times with $300 \mu \mathrm{l}$ of wash buffer. Microplates were then blocked with $200 \mu \mathrm{l}$ of assay diluent containing $10 \%$ fetal bovine serum albumin (Biochrom ${ }^{\mathrm{AG}}$, Berlin) and incubated for $1 \mathrm{~h}$ at room temperature (RT). After washing microplates 3 times with wash buffer, $200 \mu \mathrm{l}$ assay diluent was added, followed by $100 \mu \mathrm{l}$ plasma sample, $100 \mu \mathrm{l}$ standards (OptEIA, Belgium), serially diluted in $100 \mu \mathrm{l}$ assay diluent and $100 \mu \mathrm{l}$ controls and incubated for $2 \mathrm{~h}$ at RT. After washing microplates 5 times, $100 \mu \mathrm{l}$ working antibody detector (biotinylated detection antibody + streptavidinhorseradish peroxidase) was added and incubated for $1 \mathrm{~h}$ at RT. After 7 washes, $100 \mu \mathrm{l}$ substrate solution (tetramethylbenzidine, TMB, BD Biosciences, Belgium) 
was added and incubated in the dark for $30 \mathrm{~min}$ for colour development, after which $50 \mu \mathrm{l}$ stop solution $(2 \mathrm{M}$ $\mathrm{H}_{2} \mathrm{SO}_{4}$ ) was added and the plate read at $450 \mathrm{~nm}$ using a microplate reader (Biotek, UK). All assays were done in triplicate wells on the same plate and readings averaged. For TGF- $\beta$, the plasma sample was centrifuged for $2 \mathrm{~h}$ at $1800 \times g$ to release the latent TGF- $\beta 1$ into plasma and then activated through acidification with $1 \mathrm{M} \mathrm{HCl}$ at $4{ }^{\circ} \mathrm{C}$ for $60 \mathrm{~min}$ and neutralised with $1 \mathrm{M} \mathrm{NaOH}$. Cytokine concentrations of test samples were extrapolated from the optical density (OD)-concentration standard curve obtained from serial dilution of recombinant cytokine standards using GraphPad Prism 6.0 statistical package.

\section{Data analysis and management}

All numerical variables were summarized using mean and standard deviation of mean. All comparisons of categorical variables and cytokine data were analyzed using Graphpad Prism 6.0 statistical packages. Comparison of categorical variables was performed using Chi-square test at $\mathrm{P}<0.05$ significance. Deviation from normality was tested using D'Agostino and Pearson omnibus normality test of plasma cytokine data. Cytokine data was presented as medians since it did not present a normal distribution. Comparison of cytokine levels between the different groups of participants was done using nonparametric tests; Mann-Whitney $U$ and Kruskal-Wallis tests followed by Dunn's multiple comparisons test at a significant level $(\mathrm{P}<0.05$, two tailed). Correlation analysis between cytokines was performed using bivariate non-parametric Spearman's correlation rank test at a significant level $(\mathrm{P}<0.05$, two tailed).

\section{Results}

Demographic and baseline characteristics of participants A total of 60 cases and 30 healthy controls recruited at Lwala hospital were enrolled for the study after hospital microscopy diagnosis, HCT and PCR parasite species identification. The ratio of male (48) to female (42) was approximately 1:1 with an average age of $28.8 \pm 14.1$ years. Participants 18 years and above were significantly more infected than those of six and below 18 years $(P<0.05)$. There was no significant association between sex and infection status $(P>0.05)$. There were 14 early and 18 late stage HAT cases in this study with no significant difference $(\mathrm{P}>0.05)(\mathrm{Chi}$-square, $\mathrm{P}<0.05)$. Among the participants; malaria mono-infected cases were $31.1 \%$ (28/90), $18.9 \%$ (17/90) were HAT monoinfected, while $16.7 \%(15 / 90)$ had HAT and malaria co-infection and $33.3 \%(30 / 90)$ were healthy controls. Among the 32 HAT cases, 15 were positive for $P$. falciparum malaria. Therefore, the prevalence of malaria among HAT cases was $46.8 \%(15 / 32)$ (Table 1$)$.

\section{Plasma cytokine levels in HAT and/or malaria infections relative to healthy controls}

The assay was carried out for five plasma cytokines (IFN- $\gamma$, TNF- $\alpha$, IL-6, IL-10, and TGF- $\beta$ ) in HAT and/ or malaria cases and healthy controls. The detection limits for the cytokine assays (IFN- $\gamma$, TNF- $\alpha$, IL-6, IL-10, and TGF- $\beta$ ) were $8.3,9.1,3.6,4.2$, and $522.2 \mathrm{pg} /$ $\mathrm{ml}$ respectively, calculated according to Armbruster and Pry [33]. The plasma levels of IFN- $\gamma$, IL- 6 and IL-10 were higher in HAT $(72.23,55.04$ and $115.5 \mathrm{pg} /$ $\mathrm{ml})$ or malaria cases $(14.72,10.74$, and $11.44 \mathrm{pg} / \mathrm{ml})$ respectively than healthy controls $(8.612,3.513$, and $3.812 \mathrm{pg} / \mathrm{ml}$ ) (HAT significance: $\mathrm{P}<0.0001, \mathrm{P}<0.0001$,

Table 1 Demographic and baseline characteristics of participants

\begin{tabular}{|c|c|c|c|c|c|c|}
\hline \multirow[t]{2}{*}{ Characteristic } & \multicolumn{5}{|c|}{ Participant status } & \multirow[t]{2}{*}{$P$ value } \\
\hline & Malaria & HAT & HAT + malaria & Healthy controls & Total n (\%) & \\
\hline Subjects n (\%) & $28(31.1)$ & $17(18.9)$ & $15(16.7)$ & $30(33.3)$ & $90(100)$ & \\
\hline Average age & $25.1 \pm 11.1$ & $26.5 \pm 13.4$ & $22.3 \pm 14.5$ & $36.4 \pm 14.4$ & NA & \\
\hline \multicolumn{7}{|l|}{ Sex } \\
\hline Male & 15 & 9 & 10 & 14 & $48(53.3)$ & \multirow[t]{2}{*}{$>0.05$} \\
\hline Female & 13 & 8 & 5 & 16 & $42(46.7)$ & \\
\hline \multicolumn{7}{|c|}{ Age group (years) } \\
\hline $6<18$ & 4 & 5 & 5 & 1 & $15(16.7)$ & \multirow[t]{2}{*}{$<0.05^{\mathrm{a}}$} \\
\hline$\geq 18$ & 24 & 12 & 10 & 29 & $75(83.3)$ & \\
\hline \multicolumn{7}{|l|}{ HAT stage } \\
\hline Early & NA & 7 & 7 & NA & $14(47.8)$ & \multirow[t]{2}{*}{$>0.05$} \\
\hline Late & NA & 10 & 8 & NA & $18(56.2)$ & \\
\hline
\end{tabular}

HAT Human African Trypanosomiasis, NA not applicable

a Significantly higher in participants $\geq 18$ years, $6<18$; young adults, $\geq 18$; adults 
$\mathrm{P}<0.0001$ or malaria significance: $\mathrm{P}=0.0169, \mathrm{P}=0.0015$, and $\mathrm{P}=0.0288$ ) respectively. These cytokines were also significantly elevated in HAT over malaria cases $(\mathrm{P}=0.0027 ; \mathrm{P}=0.0060 ;$ and $\mathrm{P}=0.0057$, Fig. $2 \mathrm{a}, \mathrm{c}, \mathrm{d})$ respectively. Similarly, plasma TNF- $\alpha$ and TGF- $\beta$ level of HAT (35.15 and $1379 \mathrm{pg} / \mathrm{ml}$ ) or malaria (32.22 and $1434 \mathrm{pg} / \mathrm{ml}$ ), was significantly elevated over healthy controls (24.11 and $534.7 \mathrm{pg} / \mathrm{ml}$ ), (HAT significance: $\mathrm{P}=0.0052$ and $\mathrm{P}=0.0003$ or malaria significance: $\mathrm{P}=0.0103$ and $\mathrm{P}=0.0009$ ) respectively. However, no significant difference in the level of these cytokines (TNF- $\alpha$ and TGF- $\beta$ ) between HAT and malaria cases was noted $(P>0.9999)$, (Fig. 2b, e respectively, KruskalWallis, Dunn's multiple comparisons test, $\mathrm{P}<0.05$ ). The HAT and malaria co-infection expressed significantly higher median plasma cytokine level of IFN- $\gamma$, TNF$\alpha$, IL-6, IL-10, and TGF- $\beta$ (37.78, 52.72, 127.5, 205.3, and $1922 \mathrm{pg} / \mathrm{ml}$ ) respectively than healthy controls $(\mathrm{P}<0.0001$, Fig. 3) (Mann-Whitney U test, $\mathrm{P}<0.05)$.

\section{Plasma cytokine level in HAT and malaria co-infection relative to mono-infections}

The co-infected cases expressed a significantly higher plasma level of IFN- $\gamma$, IL-6, and IL-10 (37.78, 127.5, and $205.3 \mathrm{pg} / \mathrm{ml})$ than malaria $(\mathrm{P}=0.0132, \mathrm{P}<0.0001$, and $\mathrm{P}<0.0001)$, but no significant difference with HAT $(\mathrm{P}>0.9999, \mathrm{P}=0.8151$, and $\mathrm{P}=0.4213$ ) (Fig. $4 \mathrm{a}, \mathrm{c}, \mathrm{d}$ ) respectively. The plasma TNF- $\alpha$ level was significantly elevated in co-infection $(52.72 \mathrm{pg} / \mathrm{ml})$ over HAT or malaria mono-infection $(\mathrm{P}=0.0438$ or $\mathrm{P}=0.0134)$
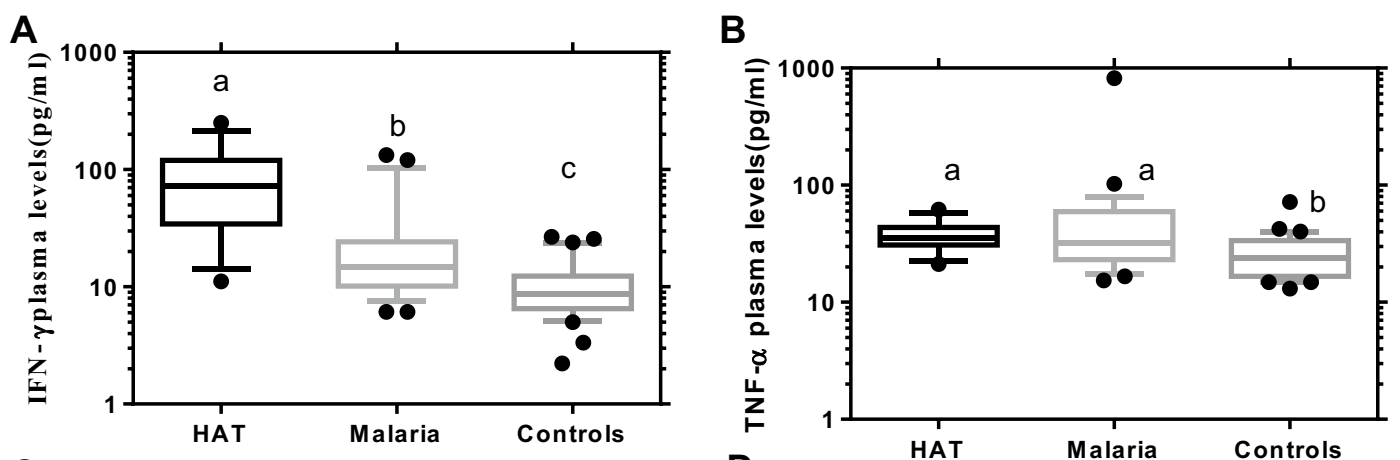

C
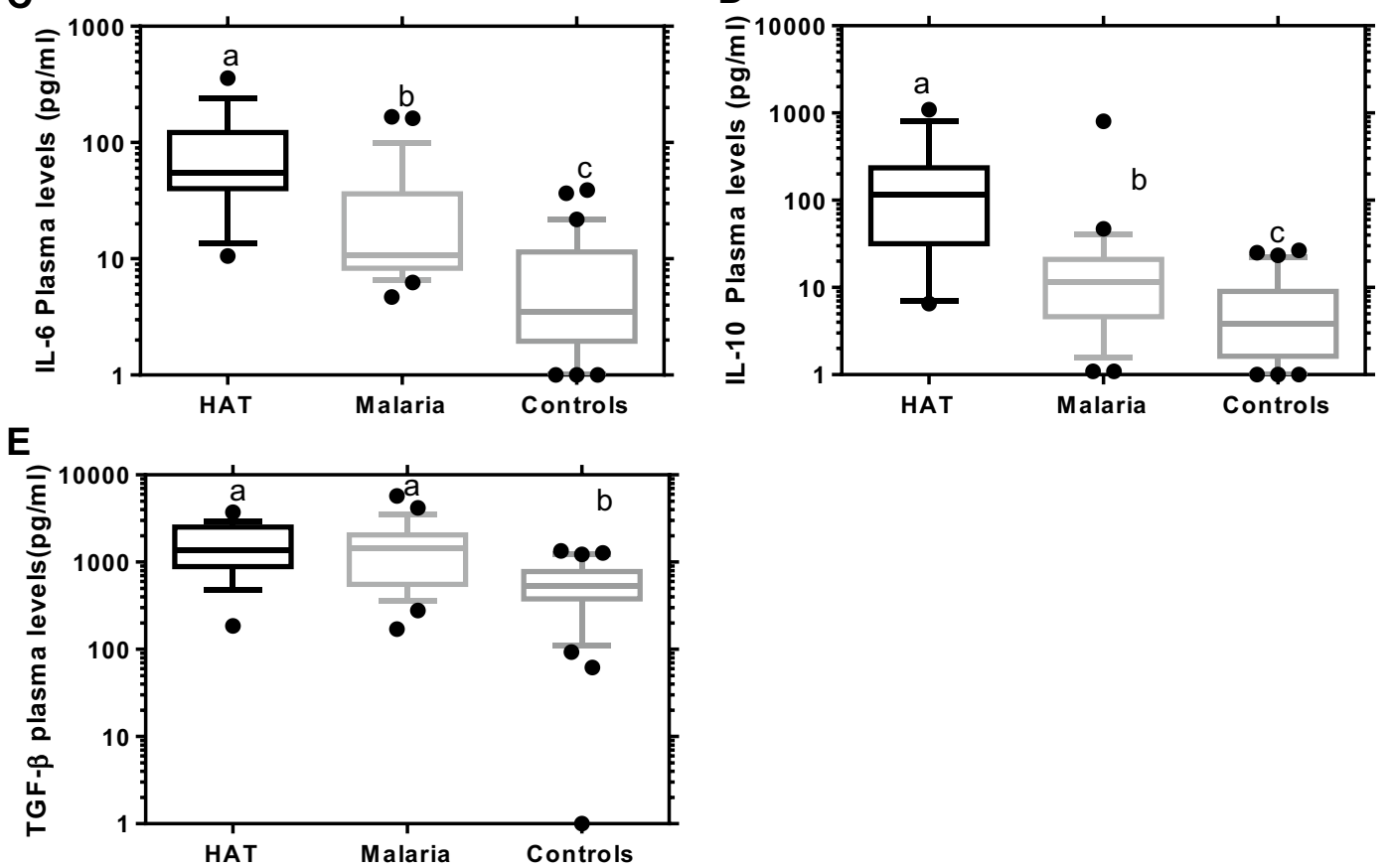

Fig. 2 Plasma cytokine levels of HAT or malaria mono-infections compared to healthy controls. Participants of HAT $(n=17)$ or malaria $(n=28)$ mono-infections and healthy controls $(n=30)$ were involved. Boxes indicate median and interquartile range, whiskers are defined as 10th-90th percentiles. Dots define outliers. Letters $(a>b>c)$ indicate level of significance between participant groups (Kruskal-Wallis, Dunn's multiple comparisons tests, $\mathrm{P}<0.05$ ) 

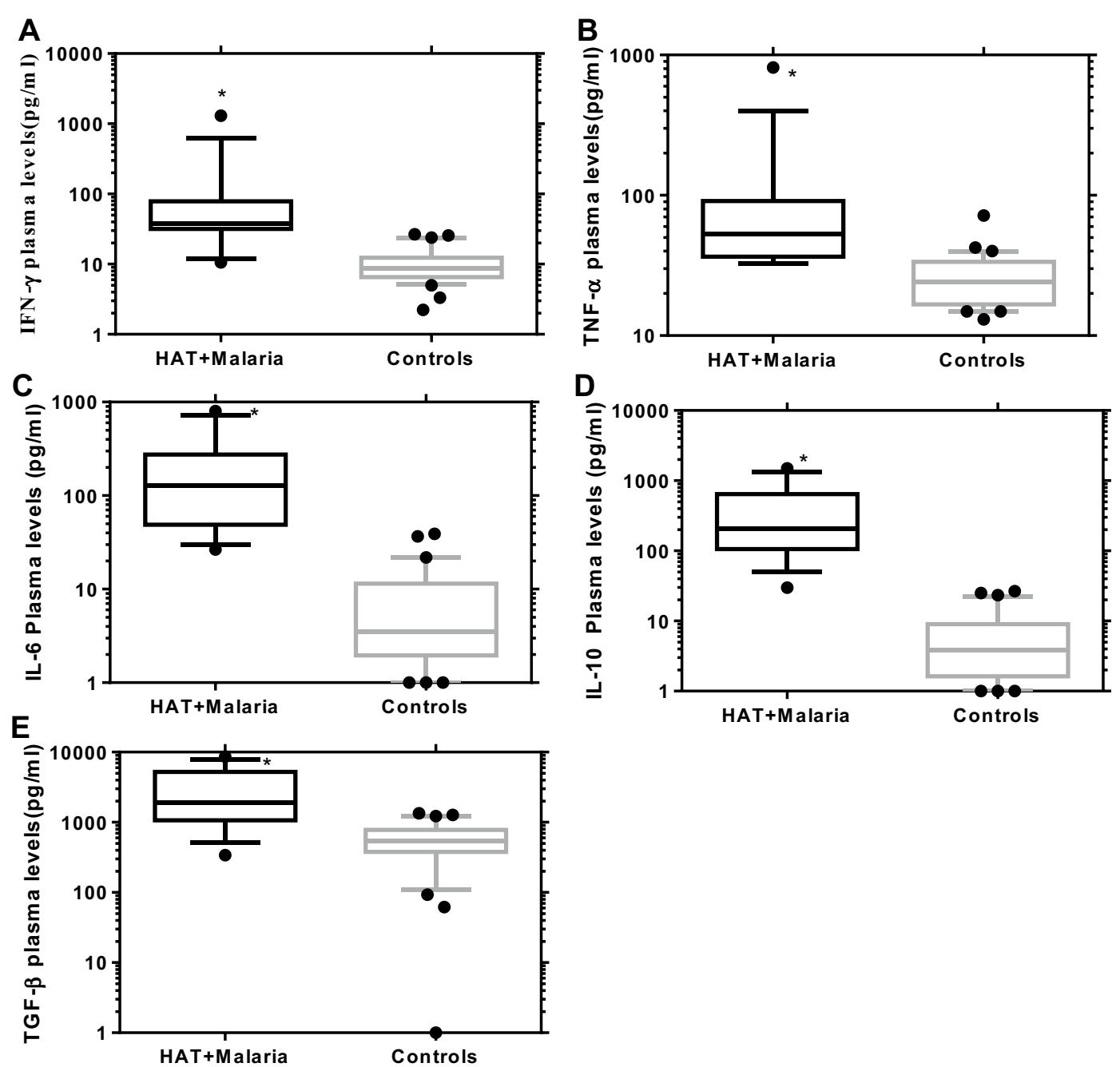

Fig. 3 Plasma cytokine levels of HAT and malaria co-infection compared to healthy controls. Participants of HAT and malaria co-infection ( $\mathrm{n}=15)$ and healthy controls $(n=30)$. Boxes indicate median and interquartile range, whiskers are defined as 10th-90th percentiles. Dots define outliers. Asterisk $\left(^{*}\right)$ indicate significant elevation in co-infected cases over healthy controls (Mann-Whitney $U$ test, $\mathrm{P}<0.05$ )

respectively (Fig. 4b). However, plasma TGF- $\beta$ level was not significantly different between co-infection $(1922 \mathrm{pg} /$ $\mathrm{ml}$ ) and mono-infection of either HAT or malaria $(\mathrm{P}=0.7494$ or $\mathrm{P}=0.2058)$ respectively (Fig. 4e) (KruskalWallis, Dunn's multiple comparisons test, $\mathrm{P}<0.05)$.

\section{Correlation between cytokine levels in HAT and malaria co-infection}

Spearman's correlation rank test was performed to investigate the association between the cytokine levels among the co-infection. Significant positive correlations were observed between IFN- $\gamma$ with TNF- $\alpha$ (Spearman $\mathrm{r}=0.847$ ) and IL-6 with IL-10 (Spearman $\mathrm{r}=0.557$ ) among co-infection. There was no significant correlation observed between the other cytokines amongst co-infection (P>0.05) (Spearman's rank correlations $\mathrm{P}<0.05,2$ tailed; Table 2; Fig. 5).

\section{Discussion}

Malaria has been reported to be prevalent among HAT cases $[2,3,34,35]$. The prevalence of $P$. falciparum malaria among $T$. $b$. rhodesiense HAT cases in this study was $46.8 \%(15 / 32)$, which is higher than $28.9 \%(70 / 242)$ earlier reported in Uganda [2]. In the same year, Kato et al. [9] reported $11 \%(6 / 55)$ malaria prevalence among HAT infected cases lower than the current findings, which were excluded from immunological studies as focused on only cytokine response among HAT cases. Studies conducted in the other countries reported 30\% HAT and malaria co-infection prevalence in Southern 
A

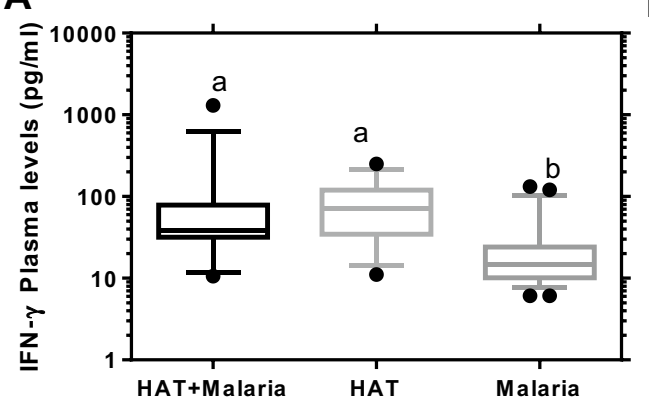

C

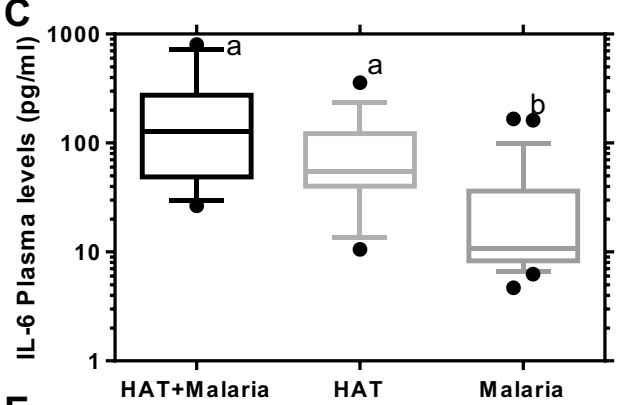

E

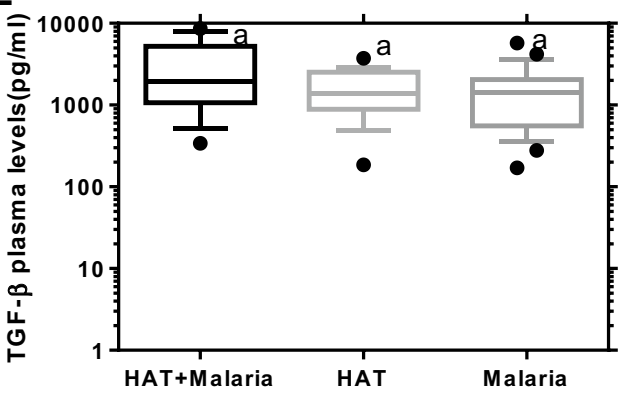

B

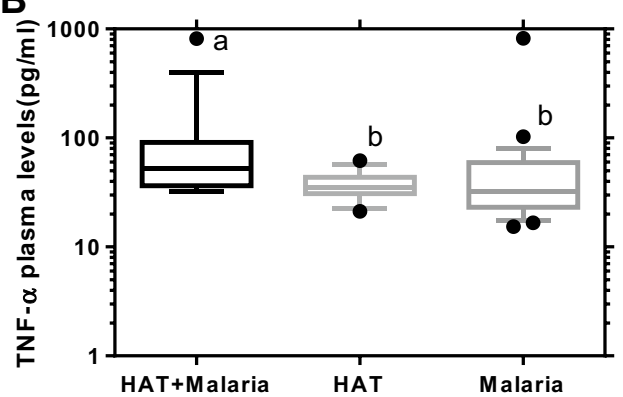

D

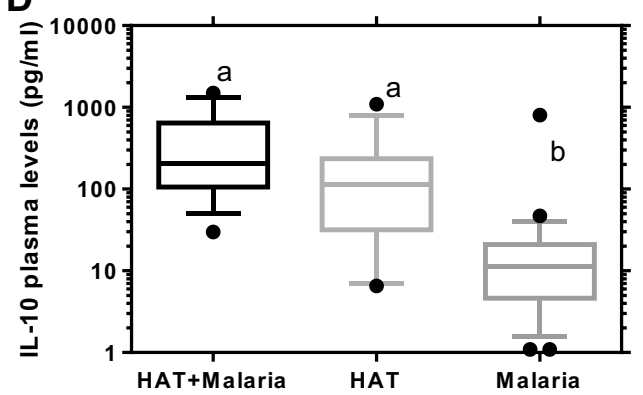

Fig. 4 Plasma cytokine levels of HAT and malaria co-infection compared to mono-infections. Participants of HAT and malaria co-infection ( $n=15)$, HAT $(n=17)$ or malaria $(n=28)$ mono-infections. Boxes indicate median and interquartile range, whiskers are defined as 10th-90th percentiles. Dots define outliers. Letters $(a>b)$ indicate significant difference between HAT and malaria co-infection compared to mono-infection (Kruskal-Wallis, Dunn's multiple comparisons test, $\mathrm{P}<0.05$ )

Table 2 Correlation coefficient between cytokine levels among HAT and malaria co-infection

\begin{tabular}{llllr}
\hline Cytokine & IFN- $\boldsymbol{\gamma}$ & \multicolumn{4}{l}{ Cytokine correlation coefficient $\mathbf{r}_{\mathbf{s}}$} \\
\cline { 2 - 5 } & TNF- $\boldsymbol{\alpha}$ & IL-6 & IL-10 & TGF- $\boldsymbol{\beta}$ \\
\hline IFN- & $0.847^{* * *}$ & 0.471 & 0.439 & 0.257 \\
TNF- $\alpha$ & & 0.431 & 0.193 & 0.312 \\
IL-6 & & & $0.557^{*}$ & -0.135 \\
IL-10 & & & & 0.014
\end{tabular}

Spearman's $\left(r_{s}\right)$ rank correlations were computed at statistical significance $\mathrm{P}<0.05^{*}$ and $\mathrm{P}<0.001^{* * *}$, negative $(-)=$ denotes negative correlation
Sudan lower than that of this study [35]. In Kenya, 100\% prevalence of HAT and malaria co-infection [3] and $79.7 \%$ in Tanzania [1], were notably higher than results of this study. The observed prevalence of co-infection in this study might have been higher, but the reported malaria self-pre-treatment, treatment of malaria before laboratory diagnosis from clinics, Ugandan government programme of free mosquito bed nets supply to every family in the region, spraying of mosquitoes and tsetse flies through the Ministry of Health and other control methods may have minimised the plasmodium and trypanosome infections [27]. 

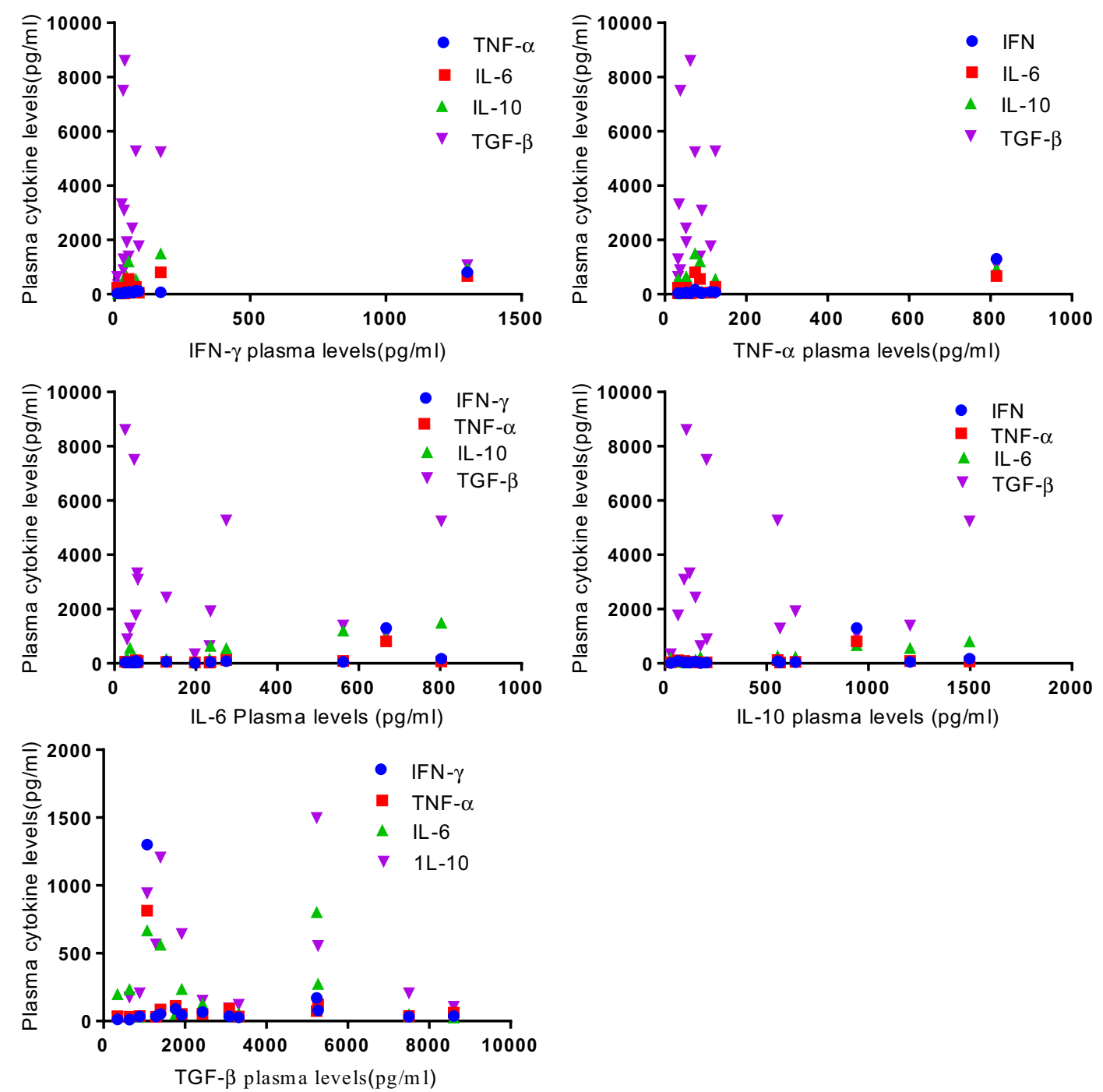

Fig. 5 Scatter plots showing correlations between plasma cytokine levels among co-infected cases $(n=15)$ (Spearman correlation significant, $P<0.05)$

Cases with HAT and/or malaria parasites responded with an overall increased secretion of plasma cytokines than healthy controls. Therefore $T . b$. rhodesiense and/or $P$. falciparum infections are associated with up-regulated cytokine responses. A significantly higher level of IFN$\gamma$, TNF- $\alpha$, IL-6, and IL-10 were expressed by co-infected cases than malaria cases, except for TGF- $\beta$ which did not vary significantly. Co-existence of HAT and malaria parasites in cases intensely changed their immunological cytokine response relative to malaria. Therefore, T. $b$. rhodesiense co-infection led to a synergistic cytokine response relative to $P$. falciparum infection in host. No significant difference was expressed between co-infected and HAT cases for cytokine IFN- $\gamma$, IL-6, IL-10 and TGF- $\beta$ levels, apart from TNF- $\alpha$ levels which were significantly higher in co-infected than mono-infected HAT cases. This indicates that $P$. falciparum infection modified the TNF- $\alpha$ level synergistically in $T$. $b$. rhodesiense infected host. The HAT cases expressed significantly higher plasma IFN- $\gamma$, IL-6 and IL-10 levels than malaria cases in this study. These findings suggest that T. $b$. rhodesiense significantly induces the host immune cytokine network more than $P$. falciparum infection. No significant difference between HAT and malaria mono-infected cases was noted for TNF- $\alpha$ and TGF- $\beta$ levels. However, no study explores the cytokine profile concentration of $T$. $b$. rhodesiense relative to $P$. falciparum in naturally infected humans. 
Cases with HAT and/or malaria parasites responded with an overall increase in secretion of pro-inflammatory cytokines (IFN- $\gamma$ and TNF- $\alpha$ ), which partially suggested the effect induced by either HAT or malaria monoinfections. This is in agreement with previous studies described in separate cases of T. $b$. rhodesiense HAT [7-9] or P. falciparum malaria [10,36]. But Da Costa et al. [37], reported that TNF- $\alpha$ level was lower in $P$. vivax malaria than healthy individuals. For trypanosome infection, it has been described that IFN- $\gamma$ is associated with the penetration of the blood brain barrier in animal models [38]. The TNF- $\alpha$ plays a key role in parasite control and infection pathology in T. brucei infections [39, 40], similar to IFN- $\gamma[13,41]$. The magnitude of TNF- $\alpha$ and IFN- $\gamma$ was associated with the progression and severity of HAT [7, 8]. Previous study by Maclean et al. [7] found higher level of plasma TNF- $\alpha$ in HAT cases from Uganda which was associated with rapid disease progression with increased disease severity compared to HAT cases from Malawi. In another HAT study however, TNF- $\alpha$ of plasma levels was low in cases and not significantly different from the controls [42]. In eastern Uganda, Tororo cases rapidly progressed to the late meningoencephalitic stage with increased disease severity associated with higher levels of plasma IFN- $\gamma$ relative to Soroti cases despite their close geographical HAT foci [8]. The elevated pro-inflammatory responses observed in plasmodium infections have been described also to protect against pre-erythrocytic stage [43-45], blood stage [43], exoerythrocytic hepatic stage development [46, 47], resolution of parasitemia [12], and acute clinical malaria manifestation $[11,48]$. Secretion of IFN- $\gamma$ and TNF- $\alpha$ in co-infected cases exhibited a significant positive correlation with each other and was significantly elevated over malaria levels. The differences in TNF- $\alpha$ levels allowed to distinguish the co-infected from both HAT or malaria mono-infected cases, indicating that both diseases synergistically contributed to its elevation. Similar observations have been reported in animal model for IFN- $\gamma$ and TNF- $\alpha$ between co- and mono-infection of trypanosome and plasmodium. [19]. The current results suggest the presence of elevated plasma IFN- $\gamma$ level of $T$. b. rhodesiense infection as compared to the P. falciparum malaria cases. The main pro-inflammatory IFN- $\gamma$ response induced by $T$. $b$. rhodesiense from natural killer (NK) and T cells may partially act as a pre-regulating activation of macrophages against plasmodium infection. This establishes protective adaptive immunity through TNF- $\alpha$ and nitric oxide impairment of hepatic stage of plasmodium $[46,49]$.

The elevation of pro-inflammatory cytokine response is regulated by anti-inflammatory cytokines. Cases with HAT and/or malaria parasites responded with an overall increased secretion of anti-inflammatory cytokines (IL-6, IL-10 and TGF- $\beta$ ). Similarly, IL-6 and IL-10 increased levels agrees with other studies in HAT $[9,42]$ or malaria infections $[36,37]$. Contrary to this study, IL-10 plasma level was significantly lower in $T$. b. gambiense HAT and P. falciparum co-infection than healthy controls. Consequently, IL-10 plasma level of HAT was not significantly affected by $P$. falciparum infection [20], in agreement with the current study. In this study, a positive correlation between IL- 6 and IL-10 elevated levels during co-infection was observed. These elevated anti-inflammatory cytokines may account for the low levels of the pro-inflammatory TNF- $\alpha$ and IFN- $\gamma$ among co-infected cases. This may suggest a regulatory response during co-infection. Therefore, trypanosome and plasmodium parasites have the ability to by-pass the immune system and interact to induce immunosuppression for their co-existence, thus enhancing their co-infection in the host [5]. Also known for its anti-inflammatory role in autoimmune conditions [17], IL-10 also possesses host protective roles against enhanced inflammation in $P$. falciparum malaria [50] and T. brucei infection [13, 41]. The role of IL-10 is to control excessive inflammation produced by the pro-inflammatory cytokines during plasmodium infection [18]. Both IL-6 and IL-10 high levels have been described to be responsible for the neuropathy decline in T. brucei [41]. Kato et al. [9] showed that plasma IL-6 was negatively associated with splenomegaly in HAT. In the current study, IL-6 and IL-10 levels were significantly elevated in HAT similar to co-infected cases over malaria mono-infection. This suggests that $T$. $b$. rhodesiense induces these anti-inflammatory cytokines that may regulate the pro-inflammatory cytokine response to $P$. falciparum during co-infection in host. The elevated TGF- $\beta$ level in HAT over healthy controls was in agreement with a study by Kato et al. [9]. At higher plasma concentrations, TGF- $\beta$ was associated with protective role in HAT cases from Malawi with slow progress to the late stage compared to Ugandan HAT cases [7]. For malaria, elevated plasma TGF- $\beta$ levels has been reported in other studies [51, 52] as observed in this study. During early malaria infection in mice, TGF- $\beta$ release is associated with slow parasite growth hence induction of protective immune response and in late infection down regulates pro-inflammatory immune responses [53]. The suppression of IFN- $\gamma$ and NO production by TGF- $\beta$ induces failure of resistance to blood stage malaria [54]. This suggests that parasite growth is associated with elevation of TGF- $\beta$ in human malaria infections. Therefore, TGF- $\beta$ modulates the Th1/Th2 immune balance through inhibition or downregulation of pro-inflammatory responses [16]. 
The sample size was small and may not be adequate to identify the effect of $T$. $b$. rhodesiense and/or $P$. falciparum infections on immune cytokine response of the host. This also limited the investigation of the cytokine levels from HAT and/or malaria infections matched by sex and age. Some pathogens for other infections might have been missed as all participants were subjected only to the routine laboratory diagnosis at the hospital associated with low sensitivity. This may explain the presence of outliers in the cytokine levels. Many HAT cases report to the hospital having been medicated or self-medicated on suspicion of malaria when it is actually HAT, since clinical symptoms are quite similar even to other disease conditions. Nevertheless, the study provides information on the immune cytokine response of $T$. $b$. rhodesiense and/or $P$. falciparum infections. Follow up studies on plasma and CSF cytokine profiles of HAT and malaria co-infections which are stratified would be appropriate. For example, cases with mild, severe, and cerebral malaria or symptomatic and asymptomatic malaria with either early or late stage HAT. This will also harmonise existing controversies about cytokines as potential disease biomarkers. Further studies about antibodies, chemokine and other biomarkers would be very important.

\section{Conclusion}

The prevalence of malaria among HAT cases was high (46.8\%). The HAT and/or malaria infection is associated with up-regulated cytokine responses. The $T . b$. rhodesiense infection significantly induced host immune cytokine response of IFN- $\gamma$, IL-6, and IL-10 more than P. falciparum infection. Malaria and HAT co-infection modified synergistically the proinflammatory (IFN- $\gamma, \mathrm{TNF}-\alpha$ ) and anti-inflammatory (IL-6, and IL-10) cytokine response than P. falciparum mono-infections. The TNF- $\alpha$ plasma level partially indicated the effect induced by mono-infections of HAT and malaria or from a synergistic effect of the co-infection which indicates a protective immunity against the parasites. The up-regulated cytokines may have adverse effects on pathogenesis, prognosis and resolution of the infections.

\footnotetext{
Abbreviations

ACT: artemisinin-based combination therapy; bp: base pairs; CNS: central nervous system; CSF: cerebrospinal fluid; DNA: deoxyribonucleic acids; ELISA: enzyme linked immuno sorbent assay; HAT: Human African Trypanosomiasis; HIV: human immunodeficiency virus; IFN- $\gamma$ : interferon-gamma; IL: interleukin; MSP: merozoite surface protein; NO: nitric oxide; OD: optical density; PCR: polymerase chain reaction; PCV: packed cell volume; SRA: serum resistance antigen; TGF- $\beta$ : transformation growth factor beta; TNF-a:
}

tumor necrosis factor alpha; T. b. r: Trypanosoma brucei rhodesiense; T. b. g: Trypanosoma brucei gambiense; UTI: urinary tract infections; WBC: white blood cell; WHO: World Health Organization.

\section{Acknowledgements}

We thank the staff at Lwala hospital for their role in collecting samples, participant enrolled into study and Welcome Trust through Afrique One for funding this study.

\section{Authors' contributions}

Designed study: JN, VPA.CDK, EM. Performed experiments: JN. Analysed data: JN, CDK, AN, VPA, EM; wrote the manuscript draft: JN, CDK, AN, VPA. All authors reviewed the manuscript. All authors read and approved the final manuscript.

\section{Funding}

Welcome Trust through Afrique One funded the research (Grant Number 087535/Z/08/A), awarded to Enock Matovu and Vincent Pius Alibu.

\section{Availability of data and materials}

The datasets used and/or analysed during the current study are available from the corresponding author on request.

\section{Ethics approval and consent to participate}

This study was ethically reviewed by Institutional Review Board (IRB) of Vector Control Division, Ministry of Health, Uganda (Ref VCD-IRC/021) and finally approved by the Uganda National Council for Science and Technology (UNCST) (Ref HS 1089). Participants were included in study after a written informed consent.

\section{Consent for publication}

Not applicable.

\section{Competing interests}

The authors declare that they have no competing interests.

\section{Author details}

${ }^{1}$ College of Veterinary Medicine, Animal Resources \& Bio-security, Makerere University, Kampala, Uganda. ${ }^{2}$ College of Natural Sciences, Makerere University, Kampala, Uganda.

Received: 18 January 2019 Accepted: 11 October 2019

Published online: 30 October 2019

\section{References}

1. Kuepfer I, Hhary EP, Allan M, Edielu A, Burri C, Johannes A. Clinical presentation of T. b. rhodesiense sleeping sickness in second stage patients from Tanzania and Uganda. PLoS Negl Trop Dis. 2011;5(3):e968.

2. Kato CD, Nanteza A, Mugasa C, Edyelu A, Matovu E. Clinical profiles, disease outcome and co-morbidities among T. b. rhodesiense sleeping sickness patients in Uganda. PLoS ONE. 2015;10(2):e0118370. https://doi. org/10.1371/journal.pone.0118370.

3. Kagira JM, Maina N, Njenga J, Karanja SM, Karori SM, Ngotho JM. Prevalence types of coinfections in sleeping sickness patients in Kenya, (2000/2009). J Trop Med. 2011. https://doi.org/10.1155/2011/248914.

4. Cheesbrough M. District laboratory practice in tropical countries. Cambridge: Cambridge University Press; 2005.

5. Cox F. Concomitant infections, parasites and immune responses. Parasitology. 2001;122(Suppl):S23-38.

6. Supali T, Verweij JJ, Eddy A, Djuardi Y, Hamid F, Kaisar MMM, Wammes LJ, Lieshout LV, Luty AJF, Yazdanbakhsh M, Sartono E. Polyparasitism and its impact on the immune system. Int J Parasitol. 2010;40:1171-6. https:// doi.org/10.1016/j.jpara.2010.05.003.

7. Maclean L, Chisi JE, Odiit M, Wendy C, Ferris V, Picozzi K, Sternberg JM. Severity of human African trypanosomiasis in East Africa is associated with geographic location, parasite genotype, and host inflammatory cytokine response profile. Infect Immun. 2004;72(12):7040-4.

8. Maclean L, Odiit M, Macleod A, Morrison L, Sweeney L, Cooper A, et al. Spatially and genetically distinct African trypanosome virulence 
variants defined by host interferon-gamma response. J Infect Dis. 2007;196(11):1620-8.

9. Kato CD, Alibu VP, Nanteza A, Mugasa CM, Matovu E. Interleukin (IL)-6 and IL-10 are up regulated in late stage Trypanosoma brucei rhodesiense sleeping sickness. PLoS Negl Trop Dis. 2015;9(6):e0003835. https://doi. org/10.1371/journal.pntd.0003835.

10. Day NPJ, Hien TT, Schollaardt T, Loc PP, Chuong LV, Chau TTH, Nguyen HP, White NJ, Hien TT, Loc PP, Mai HTH, Sinh DX, Ho M. The prognostic and pathophysiologic role of pro- and anti-inflammatory cytokines in severe malaria. J Infect Dis. 1999;180:1288-97. https://doi.org/10.1086/315016.

11. Robinson LJ, Ombrain MCD, Stanisic DI, Taraika J, Bernard N, Richards JS, Beeson JG, Tavul L, Michon P, Mueller I, Schofield L. Cellular tumor necrosis factor, gamma interferon, and interleukin- 6 responses as correlates of immunity and risk of clinical Plasmodium falciparum Malaria in children from Papua New Guinea. Infect Immun. 2009;77(7):3033-43.

12. Oyegue-liabagui SL, Bouopda-tuedom AG, Kouna LC, Nzoughe H, Tchitoula-makaya N, Pegha-moukandja I. Pro- and anti-inflammatory cytokines in children with malaria in Franceville, Gabon. Am J Clin Exp Immunol. 2017;6(2):9-20.

13. Namangala B, Noe W, De Baetselier P, Brys L, Beschin A. Relative contribution of interferon- $\gamma$ and interleukin-10 towards resistance to murine African trypanosomosis. J Infect Dis. 2001;183:1794-800.

14. Namangala B, Baetselier PDE, Beschin A. Both type-I and type-II responses contribute to murine trypanotolerance. J Vet Med Sci. 2008;71 (3):313-8.

15. Dodoo D, Omer FM, Todd J, Akanmori BD, Koram KA, Riley EM. Absolute levels and ratios of proinflammatory and anti-inflammatory cytokine production in vitro predict clinical immunity to Plasmodium falciparum malaria. J Infect Dis. 2002;185:971-9. https://doi.org/10.1086/339408.

16. Walther $\mathrm{M}$, Tongren JE, Andrews $\mathrm{L}$, Korbel $\mathrm{D}$, King $\mathrm{E}$, Fletcher $\mathrm{H}$, et al. Upregulation of TGF- $\beta$, FOXP3, and CD4+CD25+ regulatory T cells correlates with more rapid parasite growth in human malaria infection. Immunity. 2005;23:287-96. https://doi.org/10.1016/j.immuni.2005.08.006.

17. Sarciron ME, Gherardi A. Cytokines involved in toxoplasmic encephalitis. Scand J Immunol. 2000;52:534-43.

18. Ho M, Schollaardt T, Snape S, Looareesuwan S, Suntharasamai P, White NJ. Endogenous interleukin-10 modulates proinflammatory response in Plasmodium falciparum malaria. J Infect Dis. 1998;178:520-5.

19. Vaz AMR. The reciprocal influence of malaria and sleeping sickness co-infections the reciprocal influence of malaria and sleeping sickness co-infections. EMBO Young Scientists Forum. 2013 (Thesis).

20. Courtin D, Jamonneau V, Koffi M, Milet J, Sese C. Comparison of cytokine plasma levels in human African trypanosomiasis. Trop Med Int Health. 2006;11(5):647-53.

21. Fèvre EM, Picozzi K, Fyfe J, Waiswa C, Odiit M, Coleman PG, et al. A burgeoning epidemic of sleeping sickness in Uganda. Lancet. 2005;366:745-7.

22. WHO. Control and surveillance of human African trypanosomiasis: report of a WHO expert committee, vol. 984., WHO technical report seriesGeneva: World Health Organization; 2013. p. 1-250.

23. Selby R, Bardosh K, Picozzi K, Waiswa C, Welburn SC. Cattle movements and trypanosomes: restocking efforts and the spread of Trypanosoma brucei rhodesiense sleeping sickness in post-conflict Uganda. Parasite Vectors. 2013;6(1):281. https://doi.org/10.1186/1756-3305-6-281.

24. Chappuis F, Loutan L, Simarro P, Lejon V, Bu P. Options for field diagnosis of human African trypanosomiasis. Clin Microbiol Rev. 2005;18:133-46.

25. Woo PTK. The haematocrit centrifuge technique for the diagnosis of African trypanosomiasis. Acta Trop. 1970;27:384-6. https://doi. org/10.1139/z69-150.

26. Mumba Ngoyi D, Menten J, Pyana PP, Büscher P, Lejon V. Stage determination in sleeping sickness: comparison of two cell counting and two parasite detection techniques. Trop Med Int Health. 2013;18:778-82.

27. UBOS and ICF Macro. Uganda malaria indicator survey 2009. Calverton: UBOS and ICF Macro; 2010.

28. Center for Disease Control and Prevention. Guidelines for the use of antiretroviral agents in pediatric HIV infection. Center for Disease Control and Prevention: Atlanta; 1998.

29. WHO. Evidence for advocacy: key statistics on the fight against malaria. Roll back malaria partnership. 2015.

30. Maina NWN, Oberle M, Otieno C, Kunz C, Maeser P, Ndung JM, Brun R. Isolation and propagation of Trypanosoma brucei gambiense from sleeping sickness patients in south Sudan. Trans R Soc Trop Med Hyg. 2007;101:540-6.

31. Dokomajilar C, Greenhouse B, Cattamanchi CA, Parikh S, Nsobya S, Dorsey G. Protocol for genotyping P. falciparum; merozoite surface protein (Msp1), merozoite surface protein (Msp-2). 2006.

32. Chiswick EL, Duffy E, Japp B, Remick D. Detection and quantification of cytokines and other biomarkers. Methods Mol Biol. 2012;844:15-30. https ://doi.org/10.1007/978-1-61779-527-5_2.

33. Armbruster AD, Pry T. Limit of blank, limit of detection and limit of quantitation. Clin Biochem Rev. 2008;29(Suppl 1):S49-52.

34. Malele II, Kibona SN, Matemba LE, Sahani K, Swilla J, Mwalimu CD, Mayala BK, Kimaro E, Msumary C, Kalinga RB. Human African trypanosomiasis and challenges to its control in Urambo, Kasulu and Kibondo Districts, western Tanzania. Tanzan Health Res Bull. 2006;8(2):80-5.

35. Maina NW, Kagira JM, Oberle M, Ndung'u JM, Brun R. Co-infection of sleeping sickness patients with malaria and loiasis in southern Sudan. J Protozool Res. 2010;20(3):12-9.

36. Rodrigues-da-Silva RN, Lima-Junior JD, Antas PR, Baldez A, Storer FL, Santos F, Banic DM, Oliveira-Ferreira JD. Alterations in cytokines and haematological parameters during the acute and convalescent phases of Plasmodium falciparum and Plasmodium vivax infections. Mem Inst Oswaldo Cruz. 2014;109(2):154-62.

37. Guimarães da Costa A, do Valle Antonelli LR, Augusto Carvalho Costa P, Paulo Diniz Pimentel J, Garcia NP, Monteiro Tarragô A, et al. The robust and modulated biomarker network elicited by the Plasmodium vivax infection is mainly mediated by the IL-6/L-10 axis and is associated with the parasite load. J Immunol Res. 2014. https://doi. org/10.1155/2014/318250.

38. Masocha W, Robertson B, Rottenberg ME, Mhlanga J, Sorokin L, Kristensson K. Cerebral vessel laminins and IFN- $\gamma$ define Trypanosoma brucei brucei penetration of the blood-brain barrier. J Clin Investig. 2004;114(5):689.

39. Magez S, Geuskens M, Beschin A, Favero H, Verschueren H, Lucas R, De Baetselier P. Specific uptake of tumor necrosis factor-a is involved in growth control of Trypanosoma brucei. J Cell Biol. 1997;137(3):715-27.

40. Magez S, Radwanska M, Beschin A, Sekikawa K. Tumor necrosis factor alpha is a key mediator in the regulation of experimental Trypanosoma brucei infections. Infect Immun. 1999;67(6):3128-32.

41. Sternberg JM, Rodgers J, Bradley B, Maclean L, Murray M, Kennedy PGE. Meningoencephalitic African trypanosomiasis: brain IL-10 and IL-6 are associated with protection from neuro-inflammatory pathology. J Neuroimmunol. 2005;167:81-8.

42. Maclean L, Odiit M, Sternberg JM. Nitric oxide and cytokine synthesis in human African trypanosomiasis. J Infect Dis. 2001;184:1086-90.

43. Mccall MBB, Sauerwein RW. Interferon- $\gamma$-central mediator of protective immune responses against the pre-erythrocytic and blood stage of malaria. J Leukoc Biol. 2010;88(6):1131-43.

44. Perlaza B, Sauzet J, Brahimi K, Benmohamed L, Druilhe P. Interferongamma, a valuable surrogate marker of Plasmodium falciparum pre-erythrocytic stages protective immunity. Malar J. 2011. https://doi. org/10.1186/1475-2875-10-27.

45. Depinay N, Franetich JF, Mauduit M, Grüner AC, Chavatte M, Luty AJF, van Gemert G, Sauerwein RW, Siksik J, Hannoun L, Mazier D, Snounou G, Rénia L. Inhibitory effect of TNF-a on malaria pre-erythrocytic stage development: influence of host hepatocyte/parasite combinations. PLoS ONE. 2011;6(3):e17464. https://doi.org/10.1371/journal.pone.0017464.

46. Ferreira A, Schofield L, Enea V, Schellekens H, Van Der Meide P, Collins WE, Nussenzweig RS, Nussenzweig V. Inhibition of development of exoerythrocytic forms of malaria parasites by gamma-interferon. Science. 1986;232:881-4.

47. Nussler A, Pied S, Goma J, Rénia L, Miltgen F, Grau GE, et al. TNF inhibits malaria hepatic stages in vitro via synthesis of IL-6. Int Immunol. 1991;3:317-21.

48. D'Ombrain MC, Robinson LJ, Stanisic DI, Taraika J, Bernard N, Michon P, Mueller I, Schofield L. Association of early interferon-gamma production with immunity to clinical malaria: a longitudinal study among Papua New Guinean children. Clin Infect Dis. 2008:47(11):1380-7.

49. Taylor-Robinson AW. Regulation of immunity to malaria: valuable lessons learned from murine models. Parasitol Today. 1995;11(9):334-42.

50. Hunt NH, Grau GE. Cytokines: accelerators and brakes in the pathogenesis of cerebral malaria. Trends Immunol. 2003;24:491-9. 
51. Musumeci M, Malaguarnera L, Simporè J, Messina A, Musumeci S. Modulation of immune response in Plasmodium falciparum malaria: role of IL-12, IL-18 and TGF- $\beta$. Cytokines. 2003;21:172-8. https://doi. org/10.1016/S1043-4666(03)00049-8.

52. Prakash D, Fesel C, Jain R, Cazenave P, Mishra GC, Pied S. Clusters of cytokines determine malaria severity in Plasmodium falciparum-infected patients from endemic areas of Central India. J Infect Dis. 2006;194:198207. https://doi.org/10.1086/504720.

53. Omer FM, Riley EM. Transforming growth factor beta production is inversely correlated with severity of murine malaria infection. J Exp Med. 1998;188(1):39-48.
54. Tsutsui N, Kamiyama T. Transforming growth factor beta-induced failure of resistance to infection with blood-stage Plasmodium chabaudi in mice. Infect Immun. 1999:67(5):2306-11.

\section{Publisher's Note}

Springer Nature remains neutral with regard to jurisdictional claims in published maps and institutional affiliations.
Ready to submit your research? Choose BMC and benefit from:

- fast, convenient online submission

- thorough peer review by experienced researchers in your field

- rapid publication on acceptance

- support for research data, including large and complex data types

- gold Open Access which fosters wider collaboration and increased citations

- maximum visibility for your research: over $100 \mathrm{M}$ website views per year

At BMC, research is always in progress.

Learn more biomedcentral.com/submissions 